\title{
Deutsche und italienische Embleme in interkultureller Alltagskommunikation
}

Daniela Marcantonio, Università della Svizzera italiana, Faculty of Communication Sciences, Institute of Argumentation, Linguistics and Semiotics

daniela.marcantonio@usi.ch

\begin{abstract}
Gesten, als Besonderheit der menschlichen Kommunikation, haben Wissenschaftler unterschiedlicher Disziplinen immer wieder interessiert. Zahlreich sind die Studien in den Kommunikationswissenschaften, die sich mit dieser Thematik beschäftigen und die die Gestik als Teil des kommunikativen Aktes sehen. Inwiefern sind Gesten kommunikativ und kulturbezogen? Verändert sich die Gestik in interkultureller Alltagskommunikation? Ausgangspunkt der Untersuchung ist, dass Gesten häufig als ein Kulturphänomen einem Sprachumfeld zugeordnet werden. Damit stellt sich die Frage, ob die Gestik bei einem Kulturwechsel neu erlernt wird, lediglich transferiert wird oder ob aus unterschiedlichen Gesten im interkulturellen Bereich neue Gesten entstehen und vor allem, wie die Menschen, die zwischen den Kulturen leben, mit Gesten umgehen. Die zentrale These dieser Arbeit besagt, dass Gesten zwar in einer Kultur zusammen mit der Sprache erlernt werden, aber nicht ausschliesslich Bestandteil dieser Kultur sind. Nach einer umfangreichen empirischen Untersuchung, die die Variabilität und Adaption von Gesten im interkulturellen Spannungsfeld anhand von Menschen zeigt, die ihre ursprüngliche Kultur verlassen haben und sich in einer für sie neuen Kultur aufhalten, wird die These begründet: Die Übertragung von Gesten ist möglich und findet im interkulturellen Bereich auch statt. Sowohl die empirische Untersuchung in diesem interkulturellen Bereich als auch die vorgestellte theoretische Ausarbeitung sind vom Ansatz und der Umsetzung her neuartig.
\end{abstract}

\section{Keywords}

Interkulturelle Kommunikation, Semiotik, Gesten, Multimodalität, nonverbale Kommunikation

\section{Einführung in die Gestik}

Zahlreiche Studien haben gezeigt, wie bereits 1989 von Hans J. Vermeer formuliert, dass «nonverbale Kommunikation etwa $70 \%$ aller Kommunikation ausmacht; sie ist vielfach wichtiger als die verbale» (Vermeer, 1989, S. 172). Tatsächlich, wenn zwei oder mehr Leute sich unterhalten, fliessen auch extraverbale, parasprachliche und ebenso nonverbale Elemente ein (vgl. Birdwhistell, 1975; Posner, 1985). Unser Körper wird dadurch zum symbolischen Referenten für das Individuum und für die Gesellschaft. Dabei entwickelt die Person eine kinetische und proxemische Kompetenz, welche die Kontestualisierungsfähigkeit des Subjektes und den Kontextualisierungsinhalt der Nachricht in der Kollektivität repräsentiert. So können wir die Welt erkunden, in der Welt agieren und handeln, indem wir die ganze Struktur unseres Körpers aktivieren und damit sowohl Sender als auch Empfänger von Botschaften werden.

In diesem alltäglichen Kommunikationsfluss werden alle Möglichkeiten verwendet, die uns unser Körper zur Verfügung stellt, von verbalen bis $\mathrm{zu}$ nonverbalen Elementen. Diese letzte Form der Kommunikation mit Hinblick auf die Gestik hat unterschiedliche Disziplinen interessiert, die aus verschiedenen Perspektiven das Phänomen betrachtet und zahlreiche Klassifizierungen geliefert haben.

Während redebegleitende Gesten, die zur Unterstützung des Gesprochenen dienen, eine mehr oder weniger universelle Form aufweisen, scheinen dagegen Embleme (nach der Terminologie von Efron, 1941, 1972; Ekman \& Friesen, 1969, 
1972) - bedeutungstragende Gesten, wie die OK-Geste - ein Kulturspezifikum zu sein. Deshalb stimmen Psychologen, Soziologen, Anthropologen und Linguisten darin überein, dass die Gestik genauso wie die Sprache erlernt wird.

Man kann also sagen, dass die Embleme als Kulturspezifikum im Zusammenhang mit einem gewissen Sprachregister innerhalb einer bestimmten Kultur gelernt werden, also kulturbezogen sind (Morris et al., 1979; Kendon, 2004b; Efron, 1972; Ekman \& Friesen, 1969; Meo-Zilio \& Mejiá, 1980; Saitz \& Cervenka, 1972).

Aber inwiefern ist diese Art von Gesten kulturbezogen? Werden sie immer wieder vom kulturellen Sprachregister hervorgerufen? Wie wird die multimodale Alltagskommunikation im interkulturellen Bereich organisiert?

Embleme besitzen eine gewisse Autonomie in der Sprache (Ekman \& Frisen, 1972; Kendon, 1980, 1992, 2004b; Poggi, 2002) und refelktieren immer ein Konzept (Kendon, 1980). Somit können wir davon ausgehen, dass sie nicht vom Sprachregister der Kultur, in der sie erlernt wurden, abhängig sind. Warum Gesten nicht über die Grenzen der Sprachgemeinschaft hinausgehen, erklären Morris et al. (1979) mit Kultur- und Sprachbarrieren. Im Gegensatz dazu wird hier gezeigt, dass Embleme leicht in eine andere Sprachkultur transferierbar sind.

\section{2 Überblick}

Unser tägliches Leben ist voller Rituale, die unseren Tagesrhythmus bestimmen (wie aufstehen, sich waschen, frühstücken, arbeiten gehen, usw.). Als denkende, agierende und handelnde Wesen senden und empfangen wir ständig Signale und gleichzeitig setzen, produzieren und deuten wir Zeichen.

Jeden Tag interagieren wir mit Menschen, Gegenständen und Symbolen. Wir tun alles mit grösser Selbstverständlichkeit, ohne zu realisieren, wie diese Handlungen uns als kommunikative und gesellschaftliche Wesen in Anspruch nehmen.
Gemäss Watzlawick, Beavin und Jackson (1996, S. 53) ist es unmöglich, nicht zu kommunizieren. Menschen sind immer in einen Kommunikationsfluss involviert, der unsere Weltanschauung, unsere Persönlichkeit und unser Wertesystem prägt und beeinflusst.

Seit der Antike hat die Körpersprache Redner, Philosophen und Rhetoriker beschäftigt und die körperlichen Aspekte der Kommunikation finden sich bereits in der antiken Rhetorik, wo dann vor allem Gestik, Mimik und Körperhaltung eine Rolle spielten (hierzu Göttert, 1991; Maier-Eichhorn, 1989; zusammenfassende Darstellungen finden sich bei Müller, 1998, S. 25 ff.; Hübler 2001, S. 121 ff.; Kühn, 2002, S. 22 ff.).

Eine wichtige Wende in der Erforschung der nonverbalen Kommunikation im heutigen Sinne wurde mit der Arbeit von C. Darwin (1872) über den Vergleich der Ausdrucksweise von Emotionen, die sich im menschlichen und tierischen Gesicht zeigen, erreicht.

Eine erste ausführliche Auseinandersetzung mit diesem Problembereich stellt die vergleichende Arbeit von Efron (1941, 1972) dar, der die Gestik von osteuropäischen Juden und süditalienischen Einwanderern in unterschiedlichen Stadtteilen von New York City untersuchte. Aufbauend auf dieser Arbeit entwickelten vor allem Ekman und Friesen (1969) ein funktionales Klassifikationssystem von Gesten, das bis heute in der Gestikforschung verwendet wird. In diesen Studien zeigen sich klare kulturelle Unterschiede im jeweils betrachteten nonverbalen Verhalten, also der Mimik, der Gestik und der Distanzregulierung. Gleichwohl wird deutlich, dass es für alle diese Bereiche auch gleichsam anthropologische Grundlagen gibt, die für alle Menschen universell gelten, die aber in der jeweiligen Kultur unterschiedlich modifiziert und spezifiziert werden (Argyle, 1975; Scherer, 1979; Key, 1977; Ekman, 1985; Scherer \& Ekman, 1985; Siegman \& Feldstein, 1987; Burgoon et al., 1996). Dies wird vor allem in dem kulturvergleichenden Forschungsprogramm von Eibl-Eibesfeldt (1970, 1973, 1984; Eibl-Eibesfeldt et al., 1989) und von Morris (1967, 1977, 
1994; Morris et al., 1979) deutlich. Solche Resultate lassen vermuten, dass diese Art nonverbaler Urkommunikation sich dann durch kognitive Metaphorisierungs- und Metonymisierungsprozesse an ein Territorium und eine Gesellschaft kulturell gebunden ist. Daraus lässt sich folgern, dass die nonverbale Kommunikation die Basis des heutigen menschlichen Handelns ist. Unsere gestikulierende Interaktion kann also wie ein Kontinuum (Kendon, 1988; McNeill, 1992) der Hand- und Armbewegungen gesehen werden, was eine enge Beziehung zwischen dem Gesprochenen, der Körperbewegung und dem kognitiven Netzwerk des Gehirns impliziert. Eben diese letzte Implikation hat zahlreiche Psychologen dazu bewogen, Untersuchungen zu starten, welche die Gestikforschung geprägt haben.

Die Gestikforscher setzen somit ihren Schwerpunkt auf die redebegleitende Gestikulation und deren Verbindung zur Sprache, während Embleme fast nur ethnographisch untersucht werden.

Andere Untersuchungen haben das Gestenrepertoire zweier Kulturen miteinander verglichen und dabei Unterschiede festgestellt. In vielen psychologischen Studien (Petitto, 1986, 2000; Petitto et al., 2001; Bates et al., 1977, 1979, 1989; Clark, 1978; Bruner, 1975) wurde die Ontogenese der Gesten bei Kindern betrachtet. Dadurch konnten einige Fragen von semiotischem Interesse beantwortet werden, andere Fragen sind jedoch offengeblieben.

\section{Merkmale der Embleme}

Die Gestik ist in den letzten fünfzig Jahren intensiv studiert worden. Obwohl die Untersuchungen aus verschiedenen Bereichen kommen, sind sie sich alle in einem Punkt einig: Einige Gesten sind autonom und kulturell bedingt.

Wie schon erwähnt, wurden diese Gesten ursprünglich von Efron (1941, 1972) als "representing either a visual or a logical object by means of a pictorial or non-pictorial form, which has no morphological relationship to the thing represented" (Efron, 1972, S. 96) beschrieben und als «Embleme» gekennzeichnet. Dieser Begriff wurde danach von Ekman und Friesen (1972) schärfer definiert:

\begin{abstract}
Emblems are those nonverbal acts which have a direct verbal translation usually consisting of a word or two, or a phrase, for which this precise meaning is known by most or all members of a group, class, subculture or culture, which are most often deliberately used with the conscious intent to send a particular message to other persons (Ekman \& Friesen, 1972, S. 357).
\end{abstract}

Mit dieser Definition heben die Autoren drei wichtige Aspekte der emblematischen Gesten hervor: Ihre direkte sprachliche Übersetzung, sowie ihre kulturkodierte Bedeutung und ihre Intention zur Kommunikation.

Insofern ersetzen Embleme die sprachliche Äusserung, sie sind autonom, kulturbezogen, konventionell, gesellschaftlich und kommunikativ. Man kann also sagen, dass Embleme eine visuelle Metapher sind, die in einer stark kodieerten Form ein universelles Basiskonzept ausdrückt, das es ihnen erlaubt, aus den Grenzen der Kultur, in die sie eingebettet sind, herauszutreten. Daraus ergibt sich die der Arbeit zugrundeliegende These: «Gesten sind kulturbezogen, jedoch nicht kulturgebunden.»

In den meisten Arbeiten werden Embleme innerhalb einer Kultur aufgelistet, abgebildet, gedeutet und beschrieben (vgl. Marcantonio, 2008, 2016 für eine Liste der ethnographischen Arbeiten). Eine sehr gute ethnografische Untersuchung innerhalb Europas ist von Morris et al. (1979) unternommen worden. Aus dieser Untersuchung gingen 20 Schlüsselgesten hervor, die quer durch Europa zu finden waren. Morris et al. (1979) haben diese zwanzig Embleme geographisch lokalisiert, um eine Karte ihrer Verbreitung im europäischen Raum zu erstellen. Die verschiedenen Bedeutungen wurden gesammelt und damit die einzelnen Gesten beschrieben.

Während die meisten Arbeiten die interkulturellen Unterschiede der redebegleitenden Gesten, meist in Zusam- 
menhang mit einer Richtungsbeschreibung im Raum, untersucht haben (siehe Kendon, 2004a ${ }^{1}$; Müller, 1998; McNeill \& Duncan, 2000; Kita \& Ozyürek, 2003), haben nur sehr wenige Arbeiten eine kontrastive interkulturelle Untersuchung der Embleme aufgenommen, wie zum Beispiel die Studie von Efron $(1941)^{2}$, in der unterschiedliche Gesten miteinander verglichen werden, um Unterschiede und/ oder Ähnlichkeiten herauszuarbeiten. Aber meines Wissens hat sich keine Studie bis jetzt mit Emblemen beim Kulturwechsel beschäftigt. Deswegen bleiben Fragen wie die, ob die Gestik, ebenso wie die Sprache, in einer neuen Kultur neu erlernt oder einfach in diese transferiert wird und wie die Menschen mit ihr umgehen, offen.

\section{Aufbau der empirischen Untersuchung}

Gestik, Sprache oder sonstigen kulturellen Alltagsgewohnheiten der Menschen sind sowohl Teil der Mikro- wie auch der Makrokultur. Darüber hinaus dokumentieren entsprechende empirische Untersuchungen die durchschnittliche Meinung oder die Gewohnheiten ausgewählter Menschen an einem Ort und zu einer bestimmten Zeit. Gestik genauso wie Sprache, Mode und alle soziokulturelle Form der Kommunikation unterliegt zeitliche Veränderungen.

Somit werden Gesten veralten und verschwinden oder ihre Bedeutung verändern und parallel dazu entstehen neue Gesten. Trotzdem lassen sich auch immer noch die «Klassiker», wie zum Beispiel die Bündelhand für Italien, siehe Abbildung Nr. 2 aus der Kategorie "typisch italienisch"

1 Kendon (2004a) analysiert die redebegleitenden Gesten eines erwachsenen Neapolitaners im Vergleich mit einem erwachsenen Briten und stellt fest, dass es pragmatische und Gestaltungsunterschiede zwischen den beiden Kulturen gibt.

2 Kendon (2007, S. 13): "There exist remarkably few studies that can be cited that have investigated these alleged differences in any systematic way, however. The most famous study that does so, which is still the best, was first published in 1941 by David Efron.” im Paragraph 4.2, erkennen, die wiederum Merkmal für einen Kulturkreis werden. In der heutigen Welt, in welcher die Medien eine immer schnellere Kommunikation ermöglichen und auch die Menschen sich aus verschiedenen Gründen mehr von Ort zu Ort bewegen, wandert auch die Gestik und wird teilweise von neuen Kulturen aufgegriffen und dort verankert. Diese Symbole wandeln sich innerhalb des neuen Kulturkreises und werden von den Benutzern neu semantisiert und neu kodiert.

Nur wenn die emblematische Gestik als offenes und flexibles System betrachtet wird, lässt sich der Übergang von der Intrakultur zur Interkultur beobachten. Nur dann ist man imstande zu erklären, wie das Gestiksystem der Kommunikation dient und warum die gleiche Handkonfiguration in verschiedenen Länder teilweise verschiedene Bedeutungen trägt.

Also stellen sich die Fragen: Wie erlernt man die Gestik einer fremden Kultur? Wird man dabei die eigene Gestik verlieren?

Die Ergebnisse der Untersuchung zeigen, dass sich die Embleme vermischen und beiden Kulturen entnommen werden. Wenn das gleiche Konzept mit zwei unterschiedlichen Gesten zum Ausdruck kommt, wird man die Geste verwenden, die man am liebsten hat.

In der Untersuchung wurden zwei Kontrollgruppen befragt. Daraus resultierte ein Gestenglossar, welches für die Untersuchungsgruppen verwendet wurde. Das prozedere wird in den folgenden $\mathrm{Ab}$ schnitten erläutert.

\subsection{Zusammensetzung der Informanten}

Diese Analyse untersucht die Emblemdynamik im Zusammenleben zweier Kulturen: der italienischen und der deutschen. Zwischen 2002 und 2005 vier Untersuchungsgruppen in Italien und in Deutschland befragt und teilweise gefilmt (nur wenn die Person es erlaubt hat). Insgesamt haben 160 Informanten an der Studie teilgenommen, die in eine Kontroll- und Untersuchungsgruppe unterteilt wurden:

Die Kontrollgruppe (KG) besteht aus 50 Italienern, die in Italien (Rom und Sizilien) wohnen und keine Verbindung zu 
der deutschen Kultur haben und 50 Deutschen, die in Deutschland (Berlin und Stuttgart) wohnen und keine Verbindung zu der italienischen Kultur haben. Diese Informanten beider Geschlechter sind im Alter zwischen zwanzig und fünfundsechzig Jahren und haben unterschiedliches Bildungsniveau.

Mit diesen 100 Informanten wurde eine ethnographische Emblemuntersuchung der beiden Kulturkreise durchgeführt. Diese erlaubt es, Übereinstimmungen und Unterschiede der Emblemsysteme herauszufiltern.

Die Untersuchungsgruppe (UG) besteht aus 30 in Deutschland lebenden Italienern und 30 in Italien lebenden Deutschen. Die Informanten leben seit mindestens fünf Jahren im Ausland (in Italien oder in Deutschland), beherrschen sowohl die Mutter- als auch die Fremdsprache und in ihrem Alltag benutzen sie beide Sprachen. Sie wurden aber in ihrem jeweiligen Heimatland sozialisiert (das heisst, dass sie mindestens bis zu ihrem 18. Lebensjahr in Italien bzw. in Deutschland gewohnt haben).

Aufgrund der Tatsache, dass die meisten italienischen Informanten, welche in Berlin wohnen, ursprünglich aus Süditalien (Sizilien und Kampanien) stammten, habe ich dementsprechend deutsche Informanten ausschliesslich in Süditalien (Sizilien) gesucht.

Die Informanten der Untersuchungsgruppe rekrutieren sich aus dem Universitätsbereich (Dozenten und Studenten), gehören beiden Geschlechtern an und sind zwanzig bis sechzig Jahre alt.

\subsection{Methodologie der Untersuchung}

Die Vorbereitung der Untersuchung aufgrund der Beobachtung von Gesprächen, der Analyse von italienischen bzw. deutschen Filmen und der Literatur (Posner et al., 2004; Diadori, 1990; Ricci-Bitti, 1976; Poggi \& Magno Caldognetto, 1997) diente zur Festhaltung der Gesten, die am häufigsten vorkamen.

Insgesamt wurden zwanzig Embleme aus dem deutschen und dem italienischen Gestikraum abgebildet. Das erstellte Emblemcorpus wurde den Informanten der
Kontrollgruppe (Italiener in Italien und Deutsche in Deutschland) gezeigt und nach der Analyse und Systematisierung der Resultate wurden die 20 Embleme in Kategorien eingeordnet (siehe Kapitel 5) und somit der Untersuchungsgruppe gezeigt.

In vielen Untersuchungen bedienen sich der Aufzeichnung von Gesprächen in Bild und Ton um Gestik und Sprache $\mathrm{zu}$ analysieren. Dabei hat sich eine kombinierte kanal- und kodespezifische Sichtweise etabliert, die bereits in der Systematik älterer Arbeiten auftritt, dann vor allem in Arbeiten der 70er und 80er Jahre (Argyle, 1975; Scherer, 1979; Wallbott, 1982) angewandt wurde. Mit dieser Methode der Datenerhebung lassen sich die übertragungskanalspezifischen Modalitäten (wie «auditiv» oder «visuell») gut von einander unterscheiden und damit besser untersuchen.

Bezüglich der geht es darum, die aktive (bzw. bewusste) und passive Gestenkompetenz der Befragten zu testen. Hierzu bietet sich Befragungen mittels Fragebogen an. Die standardisierte Befragung mit Hilfe eines Fragebogens bringt einerseits eine hohe Vergleichbarkeit der Antworten und andererseits lassen sich die Ergebnisse quantifizieren.

Zusätzlich zum Fragebogen wurde auch ein persönliches Interview mit den Informanten geführt, das aufgrund der sprachlichen Interaktion die aktive Gestenkompetenz der Informanten zu testen erlaubt.

Die Interviews mit der Kontrollgruppe in Deutschland und in Italien benutzte eher die Methode des qualitativen Interviews, da die Gruppe nur nach der Kodierung und der Dekodierung von Emblemen gefragt wurde.

Zuerst wurden in einem kurzen small talk die Angaben zur Person gesammelt.

Anhand des Alters kann man abschätzen, wie neu oder alt eine Geste ist, wenn sie zum Beispiel von den jüngeren bzw. älteren Leuten nicht erkannt wird. Anhand der Herkunft kann man erfahren, wo eine gewisse Geste mehr Präsenz hat und anhand des Geschlechts kann man schliess- 
lich realisieren, ob eine Geste mehr von Frauen oder Männern verwendet wird.

Solche Daten sind ja wichtig für eine diatopische und eine diastratische Analyse, die aber nicht Ziel meiner Untersuchung war.

Nach dem sich kurz Kennenlernen bekamen die Informanten die Abbildungen der zwanzig Emblemen und hatten circa dreissig Sekunden bis eine Minute Zeit über jede Geste nachzudenken. Schliesslich wurden sie nach Benutzung und Bedeutung der Geste gefragt.

Die Zusammenstellung der beiden Untersuchunsgruppen hat sich als problematischer als gedacht herasugestellt, da es sich um Leute handelt, die ihres Land nicht immer freiwillig verlassen haben. Die meisten Italiener, die in Deutschland wohnen, frequentierten teilweise nur andere Italiener und haben viele Vorurteile Deutschen gegenüber. Anders sieht es bei den Deutschen aus, die in Italien leben. Viele von ihnen sind aus Lust auf etwas Neues ausgewandert und haben sich teilweise so gut integriert, dass sie die deutsche Kultur vernachlässigt haben. Deshalb musste ich Menschen aussuchen, die regelmässig nach Deutschland gefahren sind, um dort Freunde und Verwandte zu besuchen. Und bei den italienischen Informanten habe ich aufgepasst, dass diese deutschen Freunde hatten, mit welchen sie sich regelmässig trafen. Ohne diese Voraussetzungen kann es keine unvoreingenommenen Ergebnisse geben.

Diese Menschen, die zwischen den Kulturen leben, bewegen sich in einem so genannten interkulturellen Bereich. Genau in diesem Bereich stösst man auf Kulturinterferenzen, die erkennen lassen, in welcher Masse die Gestik vom Sprachregister und von der ursprünglichen Kultur unabhängig ist.

Den beiden Untersuchungsgruppen wurden Fragen nach der Alltagskommunikation gestellt (am meisten benutzte Sprache, verwendete Gestik, Erkennung von Gesten als typisch für einen Kulturkreis, Zusammenspiel mit der Sprache) und das Interview erfolgte auf Deutsch und auf Italienisch, um die Beherrschung beider Sprachen zu testen. Nachdem mit den Informanten über Gestik geredet wurde, zeigte ich ihnen die Abbildungen in Kategorien gruppiert (siehe Paragraph 5. dafür) und testete ihr passives und aktives Gestikwissen. Die Befragten mussten eine Bedeutung zu jeder Geste assoziieren und ihre Affektivität zu der Geste auf einer Skala von 1 bis 6 angeben. Das hat Informationen über die Integration der Teilnehmer geliefert. Wenn sie die Gesten der Fremdkultur als theatralisch oder ungewohnt bezeichnet haben, dann konnte ich davon ausgehen, dass sie die entsprechenden Gesten auch dann nicht verwendeten, wenn sie die Sprache dieser Kultur sprachen. Ausserdem hatten einige Informanten immer eine deutsche bzw. italienische Haltung und Gestik, unabhängig von der gesprochenen Sprache.

Im Prinzip hat auch diese letzte Beobachtung dieser Untergruppe meiner Untersuchungsgruppe meine Hypothese bestätigt, weil man feststellen konnte, dass die Körpersprache nicht vom Spracheregister abhängig und daher nicht kulturgebunden ist.

Ausserdem hat dieser abschliessende Test gezeigt, dass es in einer interkulturellen Begegnung zur Mischung der Gestik kommt. In der Tat schöpften die gut akklimatisierten Informanten die Gesten aus dem deutschen oder italienischen Kulturraum je nach Lust, Gebrauch oder Ökonomie.

\section{Zusammenstellung der Embleme}

Bei der Systematisierung der erhobenen Daten aus der Kontrollgruppe zeigte sich, dass einige Gesten von den Informanten gleich, andere unterschiedlich oder gar nicht dekodiert wurden. Somit wurden die zwanzig Embleme in vier Kategorien eingeordnet (siehe unten in diesem Paragraphen für die Abbildungen der Embleme; vgl. Marcantonio, 2016, S. 177).

, typisch italienische Embleme

, typisch deutsche Embleme

, unterschiedlich dekodierte Embleme

, gemeinsame Embleme 
Abbildung 1: $\quad$ Kategorisierte Embleme ${ }^{\mathrm{a}}$

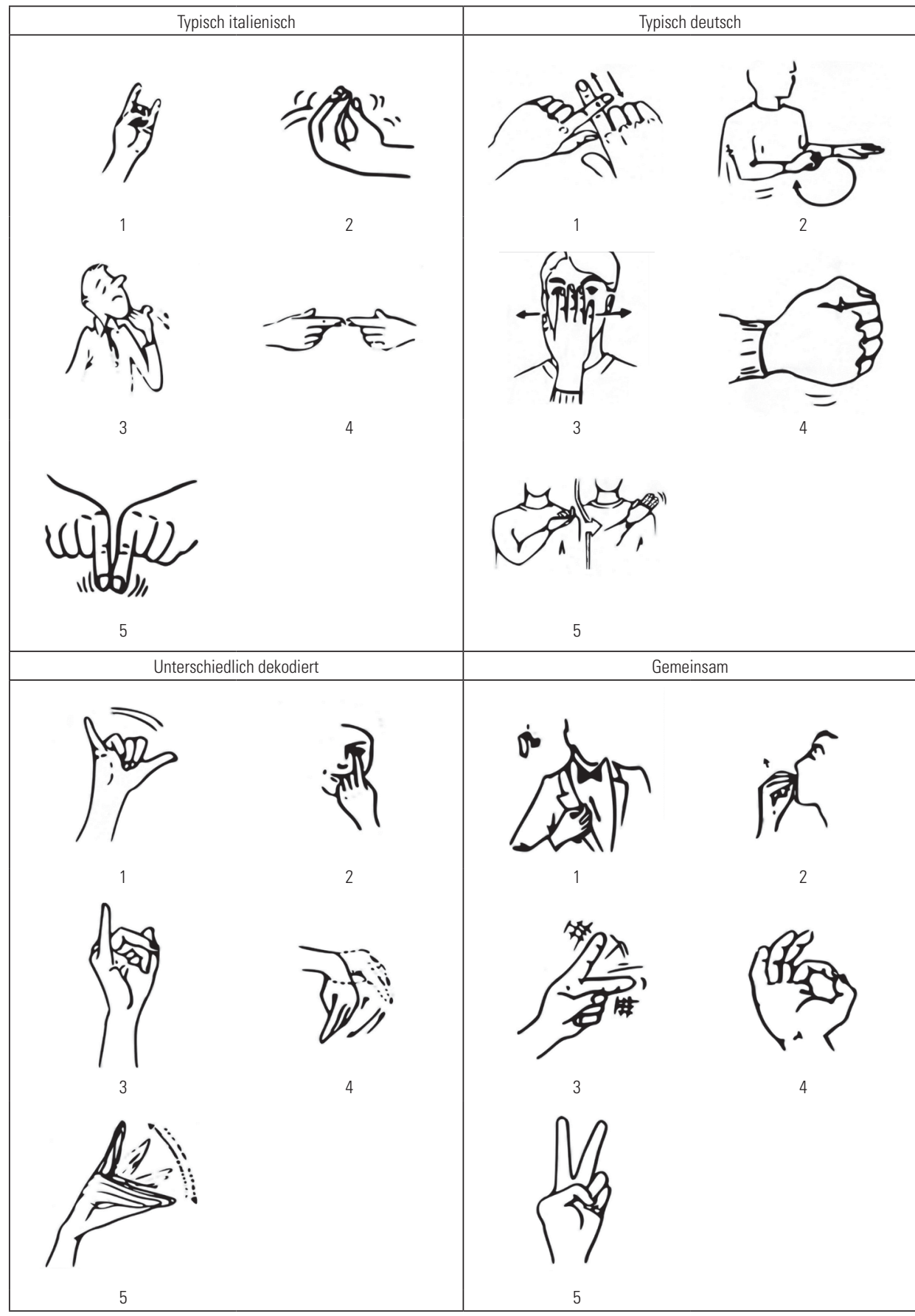

a Die folgenden Embleme bilden die zwanzig Embleme, die die Kontrollgruppe dekodiert hat. Hier werden sie so vorgestellt, wie die Untersuchungsgruppe (die Gruppe, an welcher meine Hypothese geprüft wurde) sie bekommen hat. Als Basis für die Bilder dienten die Arbeiten von Diadori (1990), Olivieri (2000), De Jorio (1832) und Romeo (1997). Insbesondere stammen die Abbildungen der Gesten: «typisch italienisch» aus Diadori (1990); "typisch deutsch» aus Marcantonio (2008, 2016); «unterschiedlich dekodiert» Nr. 1, 2 und 4 aus Diadori (1990), Nr. 3 und 5 aus Olivieri (2000); "gemeinsam» aus Diadori (1990). Die Bilder wurden teilweise leicht modifiziert. 
In den ersten zwei Kategorien sind Embleme, die von der Kontrollgruppe erkannt und dekodiert wurden, von der anderen jedoch nicht. Diese Embleme sind also der einen Kontrollgruppe «fremd» und können als «typisch» für die andere Kontrollgruppe bezeichnet werden. Das bedeutet, dass die Gesten, welche die deutschen Informanten nicht deuten konnten, die italiensichen hingegen schon, in der Kategorie «typisch italienisch» eingeordnet wurden, während die Gesten, welche die italienischen Informanten nicht deuten konnten, die deutschen aber schon, in der Kategorie «typisch deutsch» zusammengefasst wurden. Die Übereinstimmungen (Embleme, die sowohl von Deutschen als auch von Italienern erkannt wurden) genauso wie die Unterschiede, welche aus der ersten Befragung resultierten, wurden anhand ihrer Dekodierung sorgfältig verifiziert, um zu testen, wie viele Gemeinsamkeiten es zwischen den beiden Kulturkreisen gibt. Diese weitere Überprüfung ergab, dass viele Gesten, die ähnlich oder gleich aussehen, in ihrer Dekodierung (Bedeutung) jedoch voneinander abweichen. Anderseits wurde auch festgestellt, dass in einigen Fällen zwei unterschiedliche gestische Gestalten die gleiche Dekodierung besassen. Daraus ergibt sich, dass in diesen beiden Kulturkreisen die gleiche Botschaft manchmal eine andere emblematische Kodierung besitzt.

Jede Geste wurde anschliessend hinsichtlich ihrer Durchführung, ihrer Bedeutung und ihres Anwendungsfeldes beschrieben (siehe Paragraph 5.1 für ausgewählte Beispiele). Dieses ein semantisch und pragmatisch organisierte Emblemecorpus wurde den beiden Untersuchungsgruppen präsentiert.

\subsection{Ausgewählte Beispiele aus der qualitativen Analyse der Kontrollgruppe}

Wie schon oben erwähnt, hatte die Kontrollgruppe zwanzig Embleme zu dekodieren. Die Untersuchungsgruppe hat die zwanzig Embleme erhalten, nachdem sie der Kontrollgruppe vorgelegt und dort dekodiert worden waren. Basierend auf den Antworten in der Kontrollgruppe, wurden die zwanzig Embleme in vier Kategorien eingeordnet und danach der Untersuchungsgruppe gezeigt, die für das Testen der Fragestellung zusammengstellt wurde.

Unten sind einige der Embleme abgebildet. Die Beschreibungen sind auf Basis der Analyse der Daten der Kontrollgruppe erstellt worden. Danach wurden die Beschreibungen zusammen mit den Mitgliedern der Untersuchungsgruppe diskutiert, um zu testen, ob zwischen den Kulturen die Embleme noch ihre starke Kodierung behalten und wie sich die Teilnehmer der Befragung bei der Verwendung der Embleme verhalten.

Die ausgewählten Beispiele gelten jeweils als prototypisch für ihre eigene Kategorie.

\subsubsection{Prototypisches Emblem für die Kategorie «typisch italienisch»}

Kognitiv-semantisch-pragmatische Erläuterung. Die "mano a borsa" 3 (deutsch: Bündelhand) ist im Ausland das gestische Merkmal der Italiener. Tatsächlich benutzen die Italiener diese Geste mehrmals täglich. Die Frage nach Präzision, die diese Geste ausdrückt, ist auch von Morris (1994, S. 115) beobachtet worden: "Essentially this is a request for clarity. It is a 'precision posture of the hand that says, I want precise information.'” Philogenetisch, aber auch ontogenetisch gesehen, vermuten verschiedene Wissenschaftler, dass die Bündelhand ihren Ursprung bei dem Akt des Essens hat. In der Tat hat diese Geste die typische Handform, die man sieht, wenn man Kinder aber auch Primaten beim Essen beobachtet. Höchstwahrscheinlich hat schon der Homo habilis den Daumen den anderen Fingern entge-

3 So beschreibt De Jorio (1832, S. 85) die "mano a borsa" “(...) riunire in un punto tutte le dita della mano s'intende dire loro: 'riunite le vostre idee: raccogliete le tante parole in una, o in breve, in un punto e dite cosa volete! Insomma, di che si tratta”". [«Wenn man alle Finger einer Hand in einem Punkt zusammenführt, möchte man Folgendes mitteilen: (Drücke dich klar und kurz aus. Sag genau, was du willst, nämlich worum es sich handelt'», Übersetzung der Autorin]. 
Abbildung 2: Prototypisches Emblem für die Kategorie "typisch italienisch»

\begin{tabular}{l|l|l}
\hline Beschreibung der Geste & \multicolumn{2}{|c}{ Ergebnis der Umfrage (Kontrollgruppen) } \\
\hline $\begin{array}{l}\text { Die Fingerspitzen einer Hand zusammen und } \\
\text { an den Daumen führen und die Finger dabei } \\
\text { leicht krümmen. In dieser Konfiguration wird } \\
\text { die Hand aus dem Gelenk heraus hin und } \\
\text { her auf Höhe des Oberkörpers zum Sprecher } \\
\text { bewegt. }\end{array}$ & $\begin{array}{l}\text { Itienische Kontrollgruppe } \\
\text { "Che vuoi?" } \\
\text { («Was willst du?») } \\
\text { "Cosa?" } \\
\text { («Wie bitte?») } \\
\text { Anwendungsfeld: } \\
\text { Keine Bedeutung angegeben. } \\
\begin{array}{l}\text { 1. Jmd. signalisieren, dass man inn nicht } \\
\text { verstanden hat. }\end{array} \\
\text { 2. Jmd. darauf hinweisen, dass man sich } \\
\text { gestört fühlt. } \\
\text { 3. Jmd. zeigen wollen, dass man ihm nicht } \\
\text { glaubt. }\end{array}$ & $\begin{array}{l}\text { Anwendungsfeld: } \\
\text { Keine Anwendung angegeben. }\end{array}$ \\
\hline
\end{tabular}

gengesetzt, um Steine besser bearbeiten zu können. Diese Handgestalt, auch Präzisionsgriff genannt, haben Menschen also bereits vor zehntausend Jahren benutzt (Trinkaus, 1992). Neuere Studien über das Gehirn lassen vermuten, dass diese präzise Gegenüberstellung der Finger und des Daumens im Gehirn die Zellen aktivieren, die für Problemlösung, Planen, Objektmanipulation usw. zuständig sind.

Wenn diese Vermutung richtig ist, könnte man spekulieren, dass alle Menschen beim Nachdenken über Probleme spontan die Bündelhand bilden sollten. Man kann in der Tat, wenn man Talkshows oder politische Debatten - auch in Deutschland - im Fernsehen schaut, häufig diese Handform beobachten. Das bedeutet, dass Menschen unbewusst die Hand zum Bündel formen, wenn sie sich mit Themen wie Planen oder Problemlösen beschäftigen. Während in anderen
Ländern die Bündelhand, wenn überhaupt, eine redebegleitende Geste ist, ist sie in Italien ein häufig angewendetes Emblem, mit welchem man Präzision fordert. Das ist wohl der langen rhetorischen Tradition der griechischen und römischen Antike zuzuschreiben, die in Italien sehr präsent war und ist.

\subsubsection{Prototypisches Emblem für die Kategorie "typisch deutsch»}

Kognitiv-semantisch-pragmatische Erläuterung. Diese emblematische Geste, die auch in Grossbritannien bekannt ist, ist normalerweise in Deutschland eine Spottgeste, die meistens Kinder benutzen. Da die Geste in den romanischen Ländern nicht bekannt ist, vermutet man, dass es sich um ein altes, traditionsgebundenes Symbol der Germanen handelt. Bei der Befragung erkannten und deuteten

Abbildung 3: $\quad$ Prototypisches Emblem für die Kategorie "typisch deutsch»

\begin{tabular}{l|l|l}
\hline Beschreibung der Geste & \multicolumn{1}{|c}{ Ergebnis der Umfrage (Kontrollgruppen) } \\
\hline $\begin{array}{l}\text { Mit dem ausgestreckten Zeigefinger einer Hand } \\
\text { lebhaft über den Rücken des ausgestreckten Zei- } \\
\text { gefingers der anderen Hand streichen. }\end{array}$ & $\begin{array}{l}\text { Italienische Kontrollgruppe } \\
\text { Bedeutung: } \\
\text { Keine Bedeutung angegeben. } \\
\text { Anwendungsfeld: } \\
\text { Keine Anwendung angegeben. }\end{array}$ & $\begin{array}{l}\text { Bedeutung: } \\
\text { "Ätschi Bätschi!», } \\
\text { "Rüben schaben» } \\
\text { Anwendungsfeld: } \\
\text { Jemandem seine Schadenfreude } \\
\text { zeigen. Manchmal neckisch } \\
\text { gemeint. }\end{array}$ \\
\hline
\end{tabular}


Abbildung 4: Prototypisches Emblem für die Kategorie "unterschiedlich dekodiert»

\begin{tabular}{l|l|l}
\hline Beschreibung der Geste & \multicolumn{2}{|c}{ Ergebnis der Umfrage (Kontrollgruppen) } \\
\hline $\begin{array}{l}\text { Die Spitze des Zeigefingers an das untere } \\
\text { Augenlid setzen und die Haut des Augen- } \\
\text { lids so herunterziehen, dass das Auge nach } \\
\text { unten hin vergrössert erscheint. }\end{array}$ & $\begin{array}{l}\text { Italienische Kontrollgruppe } \\
\text { Bedeutung: } \\
\text { "Occhio!" («Pass auf!») } \\
\text { Anwendungsfeld: } \\
\text { Jemandem zum Aufpassen auffordern. }\end{array}$ & $\begin{array}{l}\text { Bedeutung: } \\
\text { «lch bin doch nicht blöd!», "Holzauge sei } \\
\text { wachsam!» } \\
\text { Anwendungsfeld: } \\
\text { Jemandem zeigen, dass man sich nicht ein- } \\
\text { fach hereinlegen lässt. }\end{array}$ \\
\end{tabular}

Anmerkung. Aus Marcantonio, 2016, S. 164.

alle deutschen Informanten diese Geste, aber sie gaben an, dass dieses Emblem nur von Kindern verwendet wird. Aus den Daten der deutschen Kontrollgruppe ergab sich, dass auch Erwachsene ab und zu die Spottgeste benutzen, aber mehr als Neckgeste und ohne negative Konnotation. Allgemein wird das Emblem mit Spott und/oder Schadenfreude assoziiert. Die Person, die die Geste benutzt, will damit ihre eigene Freude über den Misserfolg oder das Missgeschick (häufig selbst provoziert) einer anderen Person, die zugleich deswegen verspottet wird, ausdrücken. Der Spott kommt eben, weil die Person selbst der Grund für ihr Missgeschick ist. Daher drückt die Geste semantisch etwas anders aus, als die gewöhnlichen Spottoder Schadenfreudegesten. Der Zeigefinger, der über den anderen streicht, symbolisiert das Sich-Selbst-Verletzen (vgl. Posner et al., 2004) und wird oft von dem Spruch «da hast du dich aber geschnitten» begleitet, sozusagen als Euphemismus für «da hast du dich selbst überlistet».

\subsubsection{Prototypisches Emblem für die Kategorie «unterschiedlich dekodiert»}

Kognitiv-semantisch-pragmatische Erläuterung. Alle Gesten in der Kategorie «unterschiedlich dekodiert» werden häufig im interkulturellen Bereich missverstanden. Während die italienische Benutzung eine Botschaft für den Adressaten entschlüsselt, ist die deutsche Benutzung selbstgerichtet. Das heisst, dass die Italiener damit jemanden über eine Gefahr informieren und den Adressaten auffordern, aufmerksam zu sein. Mit dem Spruch "stai attento" (pass auf dich auf!) meinen die Italiener "tieni gli occhi aperti" (schau genau hin und lass dich nicht hereinlegen). Einen ähnlichen Spruch - «Holzauge sei wachsam» - benutzen die Deutschen. Man vermutet, dass diese Warnungen auf das Soldatenleben zurückzuführen sind. Tatsächich ist es im Krieg sehr wichtig, gute Augen zu haben und immer wachsam zu sein. Im übertragenen Sinn wird es schwierig, eine wache Person zu betrügen. Insofern will die deutsche Kodierung bedeuten: «Ich bin doch nicht blöd». Dieser Spruch wurde auch von Media Markt Deutschland lange als Werbekampagne verwendet.

Wie schon oben erwähnt, ist die Geste bei den Italienern mehr eine Warnung für jemanden. Die Geste ist oft mit dem Warnwort "occhio!" paraphrasiert. Mit dem Spruch "occhio" (Auge) drückt man kurz die Konzepte: "tieni gli occhi aperti" (schau dich gut um, schau genau hin, halte die Augen offen oder pass auf dich auf). Ein interessantes Phänomen ist, dass die deutsche Kodierung der Geste immer häufiger in Norditalien verwendet wird. Das kann zwei Ursachen haben: Die geografische Nähe und der Austausch (Zuund Abwanderung) zwischen den beiden Ländern. Die Tatsache, dass in Norditalien beide Kodierungen der "occhio" Geste (wobei die italienische Kodierung am weitesten verbreitet ist) zusammenleben, 
Abbildung 5: Prototypisches Emblem für die Kategorie "gemeinsam»

\begin{tabular}{l|l|l}
\hline Beschreibung der Geste & \multicolumn{1}{|c}{ Ergebnis der Umfrage (Kontrollgruppen) } \\
\hline $\begin{array}{l}\text { Mit dem Daumen und dem Zeigefinger einen } \\
\text { Ring bilden. Die restlichen Finger werden } \\
\text { gespreizt und weisen nach oben, wobei die } \\
\text { Handinnenfläche nach vorne zum Adressat } \\
\text { weist. }\end{array}$ & $\begin{array}{l}\text { Italienische Kontrollgruppe } \\
\text { Bedeutung: } \\
\text { "OK", "perfetto" } \\
\text { («perfekt») } \\
\text { Anwendungsfeld: } \\
\text { Jemandem zeigen, dass alles in Ordnung ist. }\end{array}$ & $\begin{array}{l}\text { Bedeutung: } \\
\text { "OK", "perfekt» } \\
\text { Anwendungsfeld: } \\
\text { Jemandem zeigen, dass alles in Ordnung ist. }\end{array}$ \\
\end{tabular}

Anmerkung. Aus Marcantonio, 2016, S. 155.

führt dazu, dass diese Geste in Italien fast immer mündlich paraphrasiert wird.

\subsubsection{Prototypisches Emblem für die Kategorie "gemeinsam»}

Kognitiv-semantisch-pragmatische Erläuterung. Das OK-Geste zusammen mit der Interjektion "okay" ist aus Nordamerika nach Europa gekommen. Die Konfiguration der Hand hat eine starke ikonische Prägung mit den beiden Buchstaben $\mathrm{O}$ und $\mathrm{K}$, indem der Zeigefinger zusammen mit dem Daumen einen Ring formen (Buchstabe $\mathrm{O}$ ), während die anderen Finger gespreizt bleiben, um das K abzubilden.

Über den Ursprung der Geste ist lange geforscht und spekuliert worden, bis der Linguist Allen Walker Read ${ }^{4}$ nach zwanzig Jahren Forschung, die sehr wahrscheinliche Theorie aufstellte, dass O.K. für die Abkürzung von "Oll Korrect" steht, die 1839 in einer Bostoner Zeitung zu lesen ist. Höchstwahrscheinlich haben die Nordamerikaner schon damals mit der Sprache gespielt und die heutige korrekte Form "all correct" eher näher an der Aussprache geschrieben. Da aber in Europa der Ringfingergeste schon lange bekannt und leicht negativ konnotiert ${ }^{5}$ ist, wundert es nicht, dass sowohl die italienische Informanten als auch die deutsche die andere «Perfekt-Geste» bevorzugen, d. h. mit

4 Vgl. dazu Metcalf (2011).

5 Vgl. Morris et al. (1979, S. 105): “The circular shape is seen as representing a body orifice, usually the anus." dem Daumen nach oben gerichtet und der Hand zur Faust geballt.

In dieser Hinsicht ist es interessant was mir verschiedene meist junge Berliner Informanten mitgeteilt haben und zwar, dass die OK-Geste im Berliner Strassenverkehr vulgär belegt ist und für den Ausruf «Arschloch» steht (Marcantonio 2016, S. 156).

\section{Analyse der Gruppendaten}

\subsection{Kontrollgruppe}

Die Daten aus der Kontrollgruppe wurden dann an der Untersuchungsgruppe getestet, die eigentlich den Kern der Untersuchung darstellt.

Die Kontrollgruppe sollte nur die Embleme kodieren und dekodieren und ihre bewusste Benutzung bestätigen. Diese Daten, die die Gestikkompetenz der Probanden darstellen, dienten als Vergleichsmuster für die Untersuchungsgruppe. Nach den Interviews mit der Kontrollgruppe war es ausserdem möglich, die zwanzig Embleme aufgrund ihrer Merkmale in Kategorien einzuordnen.

Zusammenfassend kann man eine hohe passive und aktive Kompetenz der italienischen wie der deutschen Kontrollgruppe feststellen. Allerdings ist die passive Kompetenz etwas höher als die aktive Kompetenz. Beide Kontrollgruppen haben die meisten Embleme erkannt und ohne Probleme dekodiert. Daran kann man deutlich erkennen, dass die Deutschen in 
ihrem Alltag fast soviel wie die Italiener gestikulieren. Es gibt aber mit Sicherheit Unterschiede in der Gestenfrequenz und in der Art des Gestikulierens. Aber nur die jüngeren deutschen Informanten hatten kein Problem damit einzugestehen, Gesten bewusst einzusetzen. Das ist eine interessante Tendenz, die aber in ganz Europa festzustellen ist. Höchstwahrscheinlich, weil die heutige Jugend mehr verreist und Freunde überall hat. Das bringt mit sich eine Erweiterung des Gestenrepertoirs.

Es war auch interessant festzustellen, dass die Italiener besser als die Deutschen die kulturfremden Gesten interpretiert haben. Das könnte man sich folgendermassen erklären: Da es die Italiener gewohnter sind, Gesten zu benutzen, haben sie auch mehr Phantasie, wenn sie neue Gesten deuten müssen. Vor allem viele deutschen Embleme weisen eine mehr oder weniger starke ikonische Natur auf, die die Assoziationen zu der Bedeutung vereinfacht. Italienische Embleme sind dagegen schwieriger zu dekodieren, da sie im Rahmen der Metaphorisierung einem starken Abstrahierungsprozess unterlagen. Es wäre wünschenswert, solche ersten Überlegungen durch eine gezielte Studie gründlicher zu untersuchen.

\subsection{Untersuchungsgruppen (UG)}

Die zwei Untersuchungsgruppen (Italiener in Deutschland und Deutsche in Italien) haben die Abbildungen der Embleme in vier Kategorien eingeordnet bekommen (siehe Abbildung der Kategorien in Abschnitt 5). Nach den ersten Interviews ist sofort aufgefallen, dass die Informanten eine gute passive und aktive gestische Kompetenz besitzen und dass es keine relevanten Unterschiede zu der Kontrollgruppe gibt. Einige Unterschiede sind allerdings in der Verwendung der Gestik festzustellen. Es sieht so aus, als ob die aktive Kompetenz, nämlich die bewusste Einsetzung der Gestik, bei den Italienern im Ausland höher sei, als bei den Italienern in Italien. Es wäre interessant $\mathrm{zu}$ verstehen, ob die Italiener in Deutschland sich der Gestik bewusster sind und ob sie auch mehr gestikulieren.
Die Deutschen dagegen scheinen sich in Italien gut integriert zu haben. Sie verwenden ihre Heimatgestik kaum, aber sie sind in der Lage, sie zu deuten. Beide Untersuchungsgruppen hatten eine bewusste und scharfe Einstellung zur Benutzung der Gestik und kannten sowohl die deutsche als auch die italienische Variante, die sie kontextabhängig und unabhängig vom gesprochenen Sprachregister verwenden. Daraus kann man schlussfolgern, dass die Priorität des Sprechers bei dem Konzept liegt, das er ausdrücken möchte, und dazu werden alle Mittel benutzt, die ihm zur Verfügung stehen (vgl. Marcantonio 2016, S. 212-213).

Die folgenden Diagramme stellen die aktive und passive Kompetenz der Italiener in Deutschland und der Deutschen in Italien hinsichtlich der Gesten «typisch italienisch» und «typisch deutsch» im Vergleich dar.

Diese vergleichende Analyse der relativen Häufigkeiten der ersten beiden Gestenkategorien («typisch italienisch» und «typisch deutsch») der Untersuchungsgruppe ist exemplarisch, um Rückschlüsse auf den Integrationsprozess der Informanten $\mathrm{zu}$ ziehen und um festzustellen, wie die Informanten mit ihren typischen und den ihnen fremden Gesten im Ausland umgehen (qualitative Analyse).

Anhand der erworbenen Daten, die tabellarisch und statistisch unten aufgeführt werden, kann man erkennen, dass die Untersuchungsgruppe eine gute Kompetenz in der Erkennung und Anwendung der Gestik aufweist. Fast alle Teilnehmer der Untersuchungsgruppe haben angegeben, dass sie die typisch italienischen bzw. deutschen Gesten unabhängig vom Sprachregister benutzen und sie realisieren das, weil sie von Freunden oder Verwandten darauf aufmerksam gemacht werden. All das bestätigt die Hypothese, dass die Gestik kulturbezogen, jedoch nicht kulturgebunden ist.

Interessant war es bei der Untersuchungsgruppe $\mathrm{zu}$ beobachten, dass die Informanten Lieblingsgesten - italienische und/oder deutsche - hatten, die sie immer, wenn es passte, verwendet haben. 


\section{Diagramm 1: $\quad$ Die Gestenkompetenz beider Untersuchungsgruppen im Vergleich}

Typisch italienische Gesten

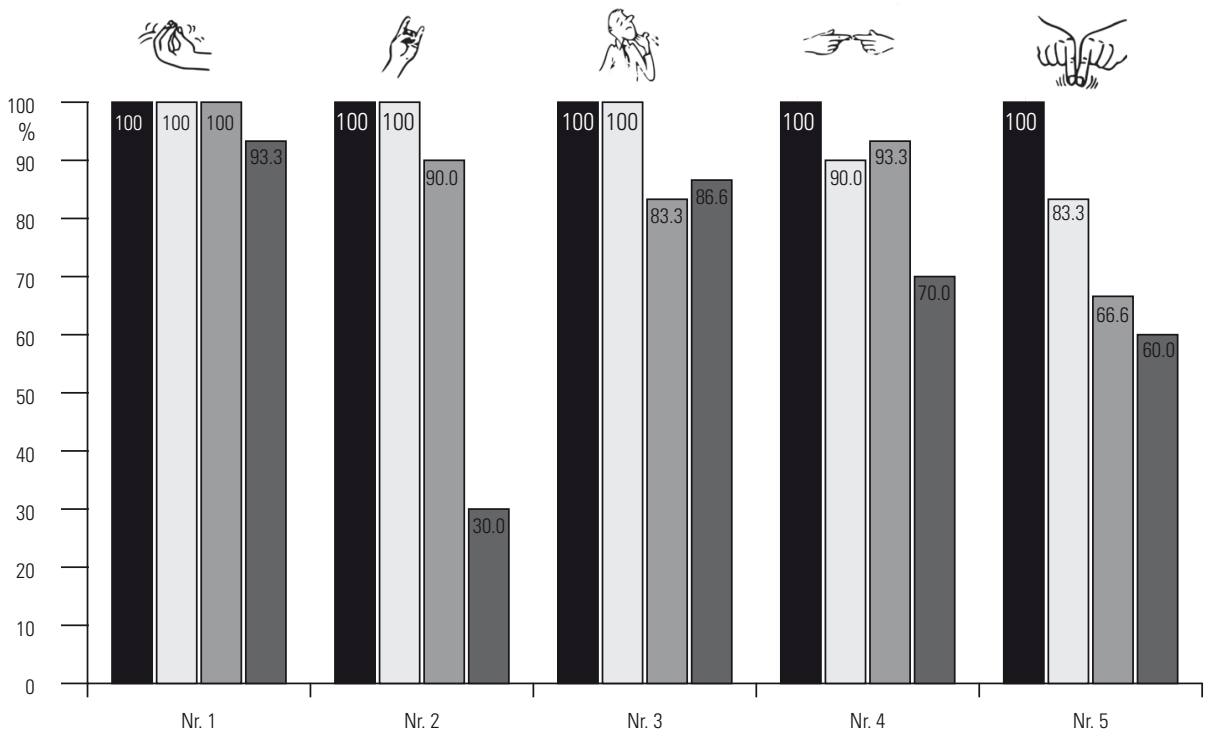

Typisch deutsche Gesten

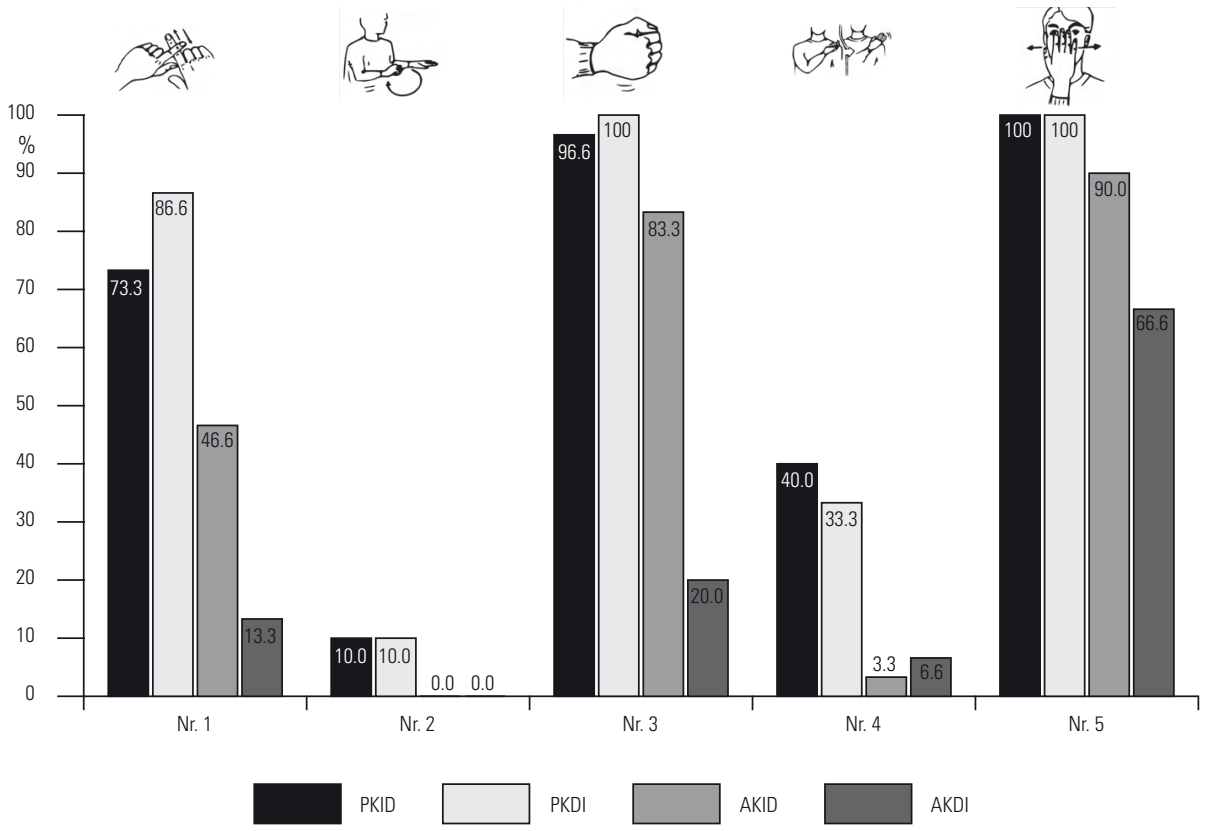

Anmerkungen: PKID=Passive Kompetenz Italiener in Deutschland, PKDI=Passive Kompetenz Deutsche in Italien, AKID=Aktive Kompetenz Italiener in Deutschland, AKDI =Aktive Kompetenz Deutsche in Italien. Aus Marcantonio, 2016, S. 208-209. 
Auf die Frage, warum sie besonders diese Geste mögen, wurde geantwortet, dass sie sich durch die Geste näher an der Landeskultur fühlten oder dass sie die Geste einfach schön, witzig oder nett fanden (vgl. Marcantonio 2016, S. 208-209). Das spricht dafür, dass die Gestik als gesellschaftlicher Faktor auch Identität und Affektivität der Person in sich trägt. Dieses Phänomen ist aber stärker bei Menschen, die im Ausland wohnen, zu beobachten. Grund dafür kann die Orientierung an die neue Kultur genauso wie die Aufbewahrung der eigenen Kultur sein. Im Ausland lebt die Person mit einer ausgeprägten kulturellen Sensibilität, deswegen sollten Variablen wie Integrationsgrad oder Kulturakzeptanz berücksichtigt werden.

\section{Zusammenfassung und Forschungsausblick}

Nach der Analyse aller Informationen, die zu Verfügung standen, konnte man schnell feststellen, wie selbstverständlich die Verwendung einiger Gesten geworden sei. Ausserdem hat man auch bemerkt, dass in der meisten Fällen die Benutzung der Gestik automatisiert worden ist, so dass die emblematische Gestik eher eine Hilfe zum Konzeptausdruck zu sein scheint, als eine Hilfe zur Unterstützung der gesprochenen Sprache. In einem interkulturellen Bereich kommt es zu einer Vermischung der Emblembenutzung je nachdem, welches Konzept die Person ausdrucken möchte und das geschieht unabhängig von der Sprache, die man in diesem Moment spricht. Das heisst, dass die Gestik sich doch nicht am Sprachregister orientiert. Daher kann man behaupten, dass die Gestenverwendung wohl kulturbezogen ist, jedoch nicht kulturgebunden. (Vgl. Marcantonio 2016, S. 213).

Darüber hinaus hat man beobachten können, dass die gestische Anpassung keine Frage der Aufenthaltslänge ist, sondern mehr eine persönliche Einstellung zu dem neuen Heimatland. Um diese Untersuchung zu erweitern aber auch zu ergänzen, wäre es wünschenswert, andere Bereiche der nonverbalen Kommunikati- on, wie Mimik und Körperhaltung, unter der Lupe zu nehmen. Eine vergleichende intrakulturelle genauso wie eine vergleichende interkulturelle Studie in dem Bereich ist immer noch ein Desiderat der nonverbalen interkulturellen Forschung.

Vor allem wünschenswert ist, wie Payrató $(1993,2003,2014)$ oft ausdrücklich geäussert hat: "Our ultimate goal is not a gestural typology per se in a purely taxonomic sense, but to describe and to explain the human communicative behavior manifested by the regular usage of conventional items with significant values and complex pragmatic functions" (Payrató 2014, S. 1479).

\section{Acknowledgements}

Inhalte dieser Arbeit wurden bereits veröffentlicht in: Marcantonio, D. (2016). Gesten im interkulturellen Vergleich. Berlin: Frank \& Timme. Der Wiederabdruck einiger Inhalte und Grafiken erfolgt mit freundlicher Genehmigung des Verlags Frank \& Timme.

\section{Literatur}

Argyle, M. (1988/1975). Bodily communication. London: Methuen.

Bates, E., Benigni, L., Bretherton, I., Camaioni, L., \& Volterra, V. (1979). The emergence of symbols: Cognition and communication in infancy. New York: Academic Press.

Bates, E., Benigni, L., Bretherton, I., Camaioni, L., \& Volterra, V. (1977). From gesture to the first word: On cognitive and social prerequisites. In L. Lewis und L. Rosenblum (Hrsg.), Interaction, conversation and the development of language (S. 247-307). New York: John Wiley.

Bates, E., Thal, D., Whitessel, K., Fenson, L., \& Oakes, L. (1989). Integrating language and gesture in infancy. Developmental Psychology, 25(6), 1004-1019.

Bausinger, H. (2000). Typisch deutsch. Wie deutsch sind die Deutschen? München: C. H. Beck.

Birdwhistell, R. L. (1975). Background considerations oft he study oft he body as a 
medium of "expression." In J. Benthall \& T. Polhemus (Hrsg.), The body as a medium of expression (S. 34-58). New York: E. P. Dutton.

Bruner, J. (1975). The ontogenesis of speech acts. Journal of Child Language, 2(1), 1-19.

Burgoon, J. K., Buller, D. B., \& Woodall, W. G. (1996, Hrsg.). Nonverbal communication. The unspoken dialogue. New York: McGraw-Hill College.

Clark, R.A. (1978). The transition from action to gesture. In A. Lock (Hrsg.), Action, gesture, and symbol. The emergence of language (S. 231-257). London: Academic Press.

Darwin, C. (1872). The expression of the emotions in man and animals. London: John Murray.

Diadori, P. (1990). Senza parole. 100 gesti degli italiani. Rom: Bonacci.

Efron, D. (1941). Gesture and environment. New York: King Crown. Republished in 1972 as Gesture, race and culture. The Hague: Mouton.

Eibl-Eibesfeldt, I., Schiefelhöven, W., \& Heeschen, V. (1989). Kommunikation bei den Eipo. Eine humanethologische Bestandaufnahme. Berlin: Reimer.

Eibl-Eibesfeldt, I. (1984). Die Biologie menschlichen Verhaltens. Grundriss der Humanethologie. München, Zürich: Blank-Media.

Eibl-Eibesfeldt, I. (1973). Der vorprogrammierte Mensch. Das Ererbte als bestimmender Faktor im menschlichen Verhalten. Wien: Orion-Heimreiter.

Eibl-Eibesfeldt, I. (1970). Ethology: The biology of behavior. New York: Holt, Rinehart and Winston.

Ekman, P. (1982). Emotions in human face. New York: Cambridge University Press.

Ekman, P. (1976). Movements with precise meanings. Journal of Communication, 26(3), 14-26.

Ekman, P., \& Friesen, W.V. (1972). Hand movements. Journal of Communication, 22(4), 353-374.

Ekman, P., \& Friesen, W.V. (1969). The repertoire of nonverbal behavior: Categories, origins, usage, and coding. Semiotica, 1, 49-98.

Göttert, K.-H. (1991). Einführung in die Rhetor$i k$. München: Fink.

Graham, J.A., \& Argyle, M. (1975). A cross-cultural study of the communication of extra-verbal meaning by gestures. International Journal of Psychology, 10(1), 57-67.

Hanna, B. E. (1996). Defining the emblem. Semiotica, 112(3/4), 289-358.

Hewes, G.W. (1974). Gesture language in culture contact. Sign Language Studies, 4, $1-34$.

Hübler, A. (2001): Das Konzept «Körper» in den Sprach- und Kommunikationswissenschaften. Basel: Franke Verlag.

Jorio de, A. (1832). La mimica degli antichi investigata nel gestire Napoletano. Neapel: Fibreno.

Kendon, A. (2007). Some topics in gesture studies. In A. Esposito, M. Bratanič, E. Keller \& M. Marinaro (Hrsg.). Fundamentals of verbal and nonverbal communication and the biometric issue (S. 3-19). Amsterdam: IOS Press.

Kendon, A. (2004a). Contrast in gesticulation. A Neapolitan and a British speaker compared. In C. Müller \& R. Posner (Hrsg.). The semantics and pragmatics of everyday gestures (Proceedings of the Berlin Conference April 1998) (S. 173-193). Berlin: Weidler.

Kendon, A. (2004b). Gesture. Visible action as utterance. Cambridge: Cambridge University Press.

Kendon, A. (1992). Some recent work from Italy on quotable gestures (emblems). Journal of Linguistic Anthropology, 2(1), 92-108.

Kendon, A. (1988). How gestures can become like words. In F. Poyatos (Hrsg.), Cross-cultural perspectives in nonverbal communication (S. 131-141). Toronto: C. J. Hogrefe.

Kendon, A. (1980), Introduction. Current Issues in the Study of "Nonverbal Communication." In A. Kendon (Hrsg.), Nonverbal communication, interaction, and gestures. Selection from semiotics (S. 1-56). The Hague, Paris, New York: Mouton Publishers.

Key, M. R. (1977). Nonverbal communication: A research guide \& bibliography. Metuchen, NJ: The Scarecrow Press, Inc.

Kirch, M. S. (1995). Deutsche Gebärdensprache. Hamburg: Buske.

Kita, S., \& Ozyürek, A. (2003). What does cross-linguistic variation in semantic coordination of speech and gesture reveal? Evidence for an interface representation of spatial thinking and speaking. Journal of Memory and Language, 48(1), 16-32. 
Kühn, C. (2002). Körpersprache. Elemente einer sprachwissenschaftlichen Explikation nonverbaler Kommunikation. Frankfurt am Main: Peter Lang.

Maier-Eichhorn, U. (1989). Die Gestikulation in Quintilians Rhetorik. Frankfurt am Main: Peter Lang.

Marcantonio, D. (2016). Gesten im interkulturellen Vergleich. Berlin: Frank \& Timme.

Marcantonio, D. (2008). Italiener in Deutschland und Deutsche in Italien: Ihre Gesten im interkulturellen Vergleich. Berlin: Technische Universität.

McNeill, D. (1992). Hand and mind. Chicago: University of Chicago Press.

McNeill, D. (Hrsg.) (2000). Language and gesture. Cambridge: University Press.

McNeill, D., \& Duncan, S. (2000). Growth points in thinking for speaking. In D. McNeil (Hrsg.), Language and gesture (S. 141-161). Cambridge: Cambridge University Press.

Metcalf, A. (2011). OK. The improbable story of America's greatest word. Oxford: Oxford University Press.

Meo-Zilio, G., Mejia, S. (1980). Diccionario de gestos. Bogotà: Instituto Caro y Cuervo

Morris, D. (1967). The naked ape. A zoologist's study of the human animal. New York: McGraw Hill.

Morris, D. (1977). Manwatching. A field-guide to human behaviour. London: Jonathan Cape.

Morris, D. (1994). Bodytalk. The meaning of human gesture. New York: Crown Trade.

Morris, D., Collett, P., Marsh P., \& O’Shaughnessy, M. (1979). Gestures. Their origin and distribution. London: Johnatan Cape.

Müller, C. (1998). Redebegleitende Gesten. Berlin: Berlin Verlag Arno Spitz GmbH.

Olivieri, F. (2000). La Gestualità dei siciliani. Palermo: Krea.

Payrató, L. (2014). Emblems, or quotable gestures: Structures, categories, and functions. In C. Müller, A. Cienki, E. Fricke, S. H. Ladewig, D. McNeill und J. Bressem (Hrsg.). Body-Language-Communication: An international handbook on multimodality in human interaction. (S. 14741481). Berlin: De Gruyter Mouton.

Payrató, L. (2003). What does the "same gesture" mean? A reflection on emblems: The need for common elicitation procedures. In M. Rector, I. Poggi und N. Trigo (Hrsg.).
Gestures, meaning and use (S. 73-81). Porto: Fernando Pessoa University Press.

Payrató, L. (1993). A Pragmatic view on autonomous gestures: A first repertoire of Catalan emblems. Journal of Pragmatics, 20, 193-216.

Petitto, L.A. (2000). On biological foundation of human language. In K. Emorrey und $\mathrm{H}$. Lane (Hrsg.). The sign of language revisited: An anthology in honour of Ursula Bellugi and Edward Klima (S. 447-471). Mahway, N. J.: Lawrence Erlbaum Assoc. Inc.

Petitto, L.A. (1986). From gesture to symbol: The relationship between form and meaning in the aquisition of personal pronouns in American Sign Language. Indiana University Linguistics Club.

Petitto, L.A., Holowka, S., Sergio, L. E., \& Ostry, D. (2001). Language rhythms in baby hand movements. Nature, 413, 35-36.

Poggi, I. (2002). Symbolic gestures; The case of the gestionary. Gesture, 2(1), 71-98.

Poggi, I. \& Magno Caldognetto, E. (1997). Mani che parlano. Padova: Unipress.

Posner, R. (1985). Nonverbale Zeichen in öffentlicher Kommunikation. Zeitschrift für Semiotik, 7(3), 235-272.

Posner, R., Krüger, R., Serenari, M., \& Noll, T. (in Vorbereitung). Berliner Lexikon der Alltagsgesten. Berlin: Weidler.

Ricci-Bitti, P.E. (1976). Communication by gestures in South and North Italians. Italian Journal of Psychology, 3, 117-125.

Romeo, O. (1997). Dizionario dei segni. Bologna: Zanichelli.

Saitz, R. L., \& Cervenka, E. J. (1972). Handbook of gesture: Colombia and the United States. The Hague: Mouton.

Scherer, K., (1979). Die Funktionen des nonverbalen Verhaltens im Gespräch. In K. Scherer, \& H. G. Wallbott (Hrsg.), Nonverbale Kommunikation. Forschungsberichte zum Interaktionsverhalten (S. 25-32). Weinheim: Beltz.

Scherer, K. R., \& Ekman, P. (Hrsg.) (1985). Handbook of methods in nonverbal behavior research. Cambridge: Cambridge University Press.

Siegman, A.W., \& Feldstein, S. (Hrsg.) (1987). Nonverbal behavior and communication. Hillsdale: Lawrence Erlbaum Associates.

Trinkaus, E. (1992). Morphological contrasts between the near Eastern Qafzeh-Skhul 
and late archaic human samples: Grounds for a behavioral difference? In T. Akazawa, K. Aoki, \& T. Kimura (Hrsg.), The evolution and dispersal of modern humans in Asia (S. 277-294). Tokyo: HokusenSha Pub. Co.

Vermeer, H. J. (1989). Wörterbücher als Hilfsmittel für unterschiedliche Typen der Translation. In F. J. Hausmann, O. Reichmann, H. E. Wiegand, \& L. Zgusta (Hrsg.), Wörterbücher. Ein internationales Handbuch zur Lexikographie (S. 171-174). Bd. 1. Berlin, New York: de Gruyter.

Wallbott, H.G. (1982). Contribution of the German "Expression Psychology" to nonverbal communication research. Part III: Gaits, gestures, and body movement. Journal of Nonverbal Behavior, 7, 20-32.

Watzlawick, P., Beavin, J.H, \& Jackson, D. D. (1996). Menschliche Kommunikation. Bern: Hans Huber. 